\title{
Hyperoxia-Exposed Lung Injury Upregulates DVL-1 Protein Expression And Activates Wnt/ $\beta$-Catenin Signaling Pathway in Newborn Rat Lung
}

Weilai Jin

Wuxi Children's Hospital

Yawen Li

Wuxi Children's Hospital

Yuting Zhu ( $\square$ tingtingyuli_911@126.com )

Wuxi Shi Ertong Yiyuan: Wuxi Children's Hospital https://orcid.org/0000-0001-7412-7272

Zhengying Li

Wuxi Children's Hospital

Le Zhang

Wuxi Children's Hospital

Fan Zhang

Wuxi Children's Hospital

Jing Chen

Wuxi Children's Hospital

Yanyu Zhang

Wuxi Children's Hospital

\section{Research}

Keywords: Hyperoxia-induced lung injury, DVL-1, Wnt/ $\beta$-catenin signaling pathway, Bronchopulmonary dysplasia (BPD)

Posted Date: October 18th, 2021

DOI: https://doi.org/10.21203/rs.3.rs-952421/v1

License: (c) (i) This work is licensed under a Creative Commons Attribution 4.0 International License. Read Full License 


\title{
Hyperoxia-exposed lung injury upregulates DVL-1 protein expression and activates Wnt/B-catenin signaling pathway in newborn rat lung
}

Weilai Jin *, Yawen Li *, Yuting Zhu*, Zhengying Li*, Le Zhang, Fan Zhang, Jing Chen, Yanyu Zhang Department of Neonatology, The Affiliated Wuxi Children's Hospital of Nanjing Medical University, Wuxi, 214023, Jiangsu, China.

*Correspondence to: Yuting Zhu, tingtingyuli_911@126.com; Zhengying E-mail: 2579434661@qq.com

\# These authors contributed equally to this work

\begin{abstract}
Background: Bronchopulmonary dysplasia (BPD) is a serious and lifelong pulmonary disease in premature neonates, which has an influence on a quarter of premature newborns. Wingless/integrated(Wnt)/ $\beta$-catenin signaling pathway affects lung cell differentiation and lung tissue structure, and is abnormal activation in the lungs of rats with pulmonary fibrosis. Method: Newborn rats were subjected to hyperoxia-exposure, histopathological changes in lung tissues were evaluated through Immunohistochemistry (IHC), Dishevelled (DVL-1) and signaling pathways were detected through western blotting and real-time PCR. Results: Contrasting with the normoxic lungs, hyperoxia-exposed lungs demonstrated larger alveoli, less alveoli and thicker alveolar septa, and the number of alveoli reduced obviously, alveoli enlarged seriously in hyperoxia group. SOD activity was decreased $\left(7^{\text {th }}\right.$ day: $P<0.05 ; 14^{\text {th }}$ day: $P<0.01)$, and MDA was increased $\left(7^{\text {th }}\right.$ day: $P<0.05 ; 14^{\text {th }}$ day: $\left.P<0.01\right)$ after hyperoxia exposure. Protein and mRNA expression levels of $\beta$-catenin, DVL-1, Ctnnbll and Cyclin D1 were upregulated by hyperoxia exposure on $7^{\text {th }}$ day $(P<0.01)$ and $14^{\text {th }}$ day $(P<0.01)$. Conclusion: We confirmed the positive role of DVL-1 and Wnt/ $\beta$-catenin signaling pathway in promoting BPD under hyperoxia conditions, and provided promising therapeutic targets in the future.
\end{abstract}

Keywords: Hyperoxia-induced lung injury; DVL-1; Wnt/ $\beta$-catenin signaling pathway; Bronchopulmonary dysplasia (BPD)

\section{Introduction}


Bronchopulmonary dysplasia (BPD) is a serious and lifelong pulmonary disease in premature neonates, which has an influence on a quarter of premature newborns [13]. Preterm newborn is related to an increased risk of long-term pulmonary problems. The pathogenesis of BPD involves a complex interaction between genetic and environmental factors. A variety of endogenous and exogenous stimulus, such as ventilator volume injury, barotrauma, hyperoxia injury, patent ductus arteriosus, result in inflammatory cascade reaction, immature lung tissue, uncontrolled pulmonary vascular development and abnormal repair of lung tissue after injury[4,5].

Dishevelled (DVL-1) had been reported to mediate three signaling pathways, including canonical Wingless/integrated(Wnt)/ $\beta$-catenin pathway, non-canonical $\mathrm{Wnt} / \beta$-catenin pathway and $\mathrm{Wnt} / \mathrm{Ca}^{2+}$ pathway[6]. In the classic canonical Wnt signaling pathway, the upregulation of DVL-1 expression leads to the overexpression of $\beta$-CATENIN protein. The accumulation of $\beta$-CATENIN protein in the cytoplasm and then promotes the transcription of $\mathrm{Wnt} / \beta$-catenin pathway downstream genes in the nucleus[7]. Recent studies showed that abnormal activation of Wnt/ $\beta$-catenin pathway caused the occurrence of pulmonary diseases $[8,9]$. In newborn lung tissue, Wnt/ $\beta$ catenin signaling pathway affects lung cell differentiation and lung tissue structure, and is abnormal activation in the lungs of rats with pulmonary fibrosis [10]. Wnt $/ \beta$-catenin pathway was activated in lung tissue in animal models of BPD and idiopathic pulmonary fibrosis, which suggested that $\mathrm{Wnt} / \beta$-catenin pathway had potential as a therapeutic target for the treatment and prevention of $\mathrm{BPD}[11,12]$.

We speculated that DVL-1 played a significant role in promoting the occurrence and development of BPD. The present study aimed to determine the role of DVL-1 and the involvement of $\mathrm{Wnt} / \beta$-catenin pathway in the occurrence and development of BPD. It was demonstrated that DVL-1 protein expression was increased and played a key role in activating Wnt/ $\beta$-catenin signaling pathway in hyperoxia-exposed newborn rat lung.

\section{Materials and methods}

\section{Reagents}

Sheep anti-rabbit horseradish peroxidase-conjugated(HRP)-conjugated secondary antibody(Cell Signaling Technology, MA, USA); RIPA lysis buffer(Cell Signaling Technology, MA, USA), BCA Protein Assay Kit, StarSignal Chemiluminescent Assay Kit (Vazyme, Nanjing, China), StarScript II First-strand cDNA Synthesis Mix With 
gDNA Remover, $2 \times$ RealStar Green Fast Mixture (with ROXII), TRIgene ( TaKaRa, Dalian, China), polyvinylidene difluoride (PVDF) membranes (Millipore, Burlington, USA); DVL-1, $\beta$-CATENIN and Cyclin D1 primary antibodies (ZENBIO biotech, Chengdu, China); GAPDH primary antibody (Goodhere biotech, Hangzhou, China); PCR primers (genscript biotech, Nanjing, China); malondialdehyde (MDA) and superoxide dismutase (SOD) assay kits ( Solarbio life sciences, Beijing, China); immunohistochemistry kit (MXB, Fujian, China).

\section{Animal procedures and treatment}

All animals' experimental procedures were approved by the Ethics Committee of Animals, The Affiliated Wuxi Children's Hospital of Nanjing Medical University. The Sprague Dawley (SD) rats were obtained from the the Nanjing Agriculture University. A total of 36 newborn SD rats were randomly selected into two groups, hyperoxia group $\left(85 \% \mathrm{O}_{2}\right.$ from the beginning of birth) and control group (normoxia, $21 \% \mathrm{O}_{2}$ ). Rats had free access to food and water. On $7^{\text {th }}$ and $14^{\text {th }}$ days after birth, nine newborn rats of two groups were anesthetized with $1 \%$ pentobarbitone by intraperitoneal injection to gather the entire lungs aseptically, respectively. The right lung was fixed with paraformaldehyde (PFA) for immunohistochemistry, the upper lobes of the left lung were used for real-time qPCR and oxidative stress index test, and the lower lobes of the left lung were used for western blotting.

\section{Oxidative stress index test}

The upper lobes of the left lung was homogenized with cold normal saline, centrifuged $\left(4{ }^{\circ} \mathrm{C}, 12500 \mathrm{~g}, 10 \mathrm{~min}\right)$, and the supernatant was collected for assays. Malondialdehyde (MDA) and superoxide dismutase (SOD) assays were performed using assay kits, according to manufacturers' instructions.

\section{Western blot analysis}

Total protein was separated from lung tissues with RIPA, and quantified with a BCA protein assay kit. After dilution with loading buffer, the separated proteins were boiled for $5 \mathrm{~min}$. Twenty micrograms of protein samples were separated using 12.5 SDS-PAGE, and electrotransferred onto polyvinylidene difluoride membranes. The membranes were probed overnight at $4{ }^{\circ} \mathrm{C}$ with primary antibodies against DVL- 
1(1:1000), $\beta$-CATENIN(1:1000), CYCLIN D1(1:1000) and GAPDH(1:5000) proteins, subsequently hybridized with horseradish peroxidase-conjugated secondary antibodies for $2 \mathrm{~h}$ at room temperature. The protein bands were detected using an ECL advanced system (Millipore, Burlington, USA) and quantified using Photoshop software.

\section{Immunohistochemistry (IHC)}

A-fixed lung tissues were dehydrated, vitrified, embedded in paraffin, fixed, and cut into $5 \mu \mathrm{M}$ thick sections, which were then fixed in a $60^{\circ}$ Coven for $4 \mathrm{~h}$. Sections were dewaxed with dimethylbenzene, hydrated with gradient alcohol according to the manufacturer's instructions, and treated with $3 \% \mathrm{H}_{2} \mathrm{O}_{2}$ to block endogenous peroxidase activity. Treated sections were placed into an EDTA-Tris buffer solution and microwaved for $20 \mathrm{~min}$, blocked with serum, and incubated overnight at $4{ }^{\circ} \mathrm{C}$ with DVL-1 primary antibody(1:200 dilution), $\beta$-CATENIN primary antibody(1:200 dilution) and CYCLIN D1 primary antibody (1:200 dilution). After sequential incubation with a biotin-labeled secondary antibody and streptavidin-peroxidase, sections were developed using 3,3'-diaminobenzidine (DAB), dehydrated. IHC score was determined semi-quantitatively by multiplication of the positive fraction with the grayscale value according to the following system: a) positive fraction was categorized as 0 , no staining; $1+, \leq 10 \% ; 2+,>10 \%,<50 \% ; 3+, \geq 50 \%$. b) grayscale value was as 0 , no staining; 1 , weaker than, or the same as that in lung; 2 , more intense than the staining in lung. IHC score of more than 2 which is, therefore, intensity score of more than 2 , was defined as positive.

\section{Real-Time Polymerase Chain Reaction (RT-PCR)}

Lung RNA was extracted using Trigene reagent according to the manufacturer's instructions. The purified mRNA was reverse transcribed into cDNA using PrimeScript ${ }^{\mathrm{TM}}$ RT reagent Kit with gDNA Eraser, and real-time PCR was performed using TB Green ${ }^{\circledR}$ Premix Ex Taq ${ }^{\text {TM }}$ II (Tli RNaseH Plus). Data were standardized to the endogenous expression of GAPDH. The sequences of the primers are listed in Table 1. Real-time PCR was performed according to the method provided by QuantStudio 3 (Applied Biosystems, USA) in a $20 \mu \mathrm{l}$ volume using $1 \mu \mathrm{l}$ cDNA, $1 \mu \mathrm{l}$ forward primer, 1 $\mu 1$ reverse primer, and $10 \mu \mathrm{l} 2 \times$ RealStar Green Fast Mixture (with ROX II). The thermal cycling conditions were as follows: Stage $1=1$ cycle, 30 s at $95^{\circ} \mathrm{C}$; Stage $2=$ 
45 cycles, $10 \mathrm{~s}$ at $95^{\circ} \mathrm{C}, 30 \mathrm{~s}$ at $60^{\circ} \mathrm{C}$; Stage $3=1 \mathrm{cycle}, 15 \mathrm{~s}$ at $95^{\circ} \mathrm{C}, 1 \mathrm{~min}$ at $60^{\circ} \mathrm{C}$.

\section{Statistical Analysis}

The experimental data were expressed as mean \pm standard deviation $(\mathrm{SD})$ values. Statistical analysis was conducted by SPSS 21.0 software (SPSS Inc., Chicago, IL, USA). One-way analysis of variance for multiple-group comparisons were performed with GraphPad Prism 8 (GraphPad Software, San Diego, USA). $P$ value of $<0.05$ was considered statistically significant.

\section{Results}

\section{Hyperoxia-induced pathological changes in the lung}

In the present study, rats exposed to $85 \% \mathrm{O}_{2}$ were inhibited alveolar development, which was correlated with the pulmonary morphological changes of BPD (Figure 1). On the 7th day, the alveoli of hyperoxia-induced rats were separated irregularly and the terminal air sacks were enlarged. Contrasting with the normoxic lungs, hyperoxiaexposed lungs demonstrated larger alveoli, less alveoli and thicker alveolar septa. As increasing duration of the hyperoxic exposure, the differences between the two groups increased significantly. On the $14^{\text {th }}$ day, the number of alveoli reduced obviously, alveoli enlarged seriously in hyperoxia group.

In hyperoxia group, IHC scores of $\beta$-CATENIN, DVL-1 and CYCLIN D1 were significantly higher than those of control group on $7^{\text {th }} \operatorname{day}(P<0.01)$, and were also significantly higher on $14^{\text {th }}$ day than those on $7^{\text {th }}$ day $(P<0.01)$ (Figure 1 , Table 2$)$. SOD is the main antioxidant enzyme, MDA is an end products of membrane lipid peroxidation, used as an indicator of cell oxidation. We found that SOD activity was decreased $\left(7^{\text {th }}\right.$ day: $P<0.05 ; 14^{\text {th }}$ day: $\left.P<0.01\right)$, and MDA was increased $\left(7^{\text {th }}\right.$ day: $P<$ $0.05 ; 14^{\text {th }}$ day: $\left.P<0.01\right)$ after hyperoxia exposure, as shown in Table 3.

\section{Hyperoxia-exposure upregulated DVL-1 protein expression and activated Wnt/ß- catenin signaling pathway in newborn rat lung}

As shown in Figure 2, compared to control group, the protein expression levels of DVL-1 were upregulated after hyperoxia exposure on $7^{\text {th }}$ day and $14^{\text {th }}$ day $(P<0.01)$, but there was no significant difference after hyperoxia exposure on $3^{\text {rd }}$ day. The protein expression levels of $\beta$-CATENIN, CTNNBL1 and CYCLIN D1 were upregulated 
after hyperoxia exposure on $3^{\text {rd }}$ day $(P<0.01), 7^{\text {th }}$ day $(P<0.01)$ and $14^{\text {th }}$ day $(P<0.01)$.

The result of Real-time PCR analysis indicated that the mRNA expression levels of $\beta$-catenin, DVL-1, Ctnnbl1 and Cyclin D1 were upregulated by hyperoxia exposure on $7^{\text {th }}$ day $(P<0.01)$ and $14^{\text {th }}$ day $(P<0.01)$.

\section{Discussion}

It is important to explore the mechanism of lung injury caused by hyperoxia for guiding clinical prevention and treatment of BPD. BPD is a chronic lung disease which is characterized by dysplasia of pulmonary alveoli and pulmonary microvascular. In the present study, we found that alveolar wall was ruptured after hyperoxia exposure continuously, and simplified structure became more obvious with the extension of hyperoxia exposure time. The evaluation of lung development results showed that the hyperoxia-exposure affected the development of the alveolar, angiogenesis and lung, ultimately induced BPD.

DVL-1 is the crucial regulator of $\mathrm{Wnt} / \beta$-catenin signaling pathway, which associates diverse arrays of biologic processes such as organogenesis, tissue homeostasis, and pathogenesis of many human diseases. In the genomes of higher organism (including mammals), three genes encoding isoforms of Dishevelled (DVL11, DVL-12, and DVL-13) are present. Canonical Wnt/ $\beta$-catenin signaling pathway was most sensitive to changes in the abundance of either DVL-13 or DVL-11[13]. Previous studies have confirmed the role of DVL-1 family proteins in promoting the occurrence and development of lung tumors. Coexpression of DVL-1 and IQ-domain GTPase-activating protein 1 (IQGAP1) in the cytoplasm and nucleus was correlated with poor prognosis of non-small cell lung cancer, and coexpression in nucleus might play a critical role in the activation of canonical Wnt/ $\beta$-catenin signaling Wnt pathway[14]. Phosphorylated Dishevelled-2 (DVL-1-2) protein were significantly higher in cisplatin resistant A549 cells compared with that in A549 cells[15]. However, there has been no research on the mechanism of DVL-1's involvement in hyperoxiainduced lung injury or BPD. Our study elucidates that increased DVL-1 expression was correlated with the development of BPD. DVL-1 protein is highly expressed in hyperoxia-induced lung injury, and not in the normal rat alveolar and bronchial epithelia.

Recent studies have shown that Wnt/ $\beta$-catenin signaling pathway plays an extremely important role in lung development, the occurrence and development of lung 
diseases [16-18]. Transforming growth factor- $\beta$ upregulates canonical WNT signaling and inhibits the peroxysome proliferator activated receptor gamma (PPAR $\gamma$ ). The absence or a decrease in $\mathrm{Wnt} / \beta$-catenin signaling during the canalicular stage of pulmonary development, partly related to inflammatory processes, severely affects the developmental processes during the subsequent saccular and alveolar stages. PPAR $\gamma$ stimulates transdifferentiation of myofibroblasts into lipofibroblasts, which helps normal alveolarization. Importantly, hypoxia and hyperoxia promote upregulation of the canonical WNT/ $\beta$-catenin system as well as TGF- $\beta$ accompanied by downregulation of PPAR $\gamma$ [18]. The administration of PPAR $\gamma$ agonist, rosiglitazone, has been shown to prevent hyperoxia-induced molecular and morphological changes in a rat model [19]. In the present study, the protein expression levels of $\mathrm{Wnt} / \beta$-catenin pathway members were increased in the hyperoxia group on $7^{\text {th }}$ and $14^{\text {th }}$. Aberrant activation of $\mathrm{Wnt} / \beta$-catenin signaling pathway induced to heterotopic differentiation of alveolar, increased alveolar volume, reduced alveolar number and resulted in simple pulmonary structure.

In the conclusion, our findings implicated that hyperoxia-exposure resulted that the number of alveoli reduced obviously, alveoli enlarged seriously in newborn rat lung tissues. Hyperoxia-exposure upregulated the protein expression levels of DVL-1 and activated $\mathrm{Wnt} / \beta$-catenin signaling pathway. These findings demonstrated the positive role of DVL-1 and Wnt/ $\beta$-catenin signaling pathway in promoting BPD under hyperoxia conditions, and provided promising therapeutic targets in the future.

\section{Acknowledgments}

Funding: The authors acknowledge support from theWuxi Key Medical Disciplines (Grant No. ZDXK12), the Wuxi Health Bureau Young Project(Grant number: Q2017016), the Wuxi Taihu Lake Talent Plan Top Talents Project(Grant number: HB2020083), Top Talent Support Program for young and middle-aged people of Wuxi Health Committee (Grant No.BJ2020089).

\section{Footnote}

Conflicts of Interest: All authors have completed the ICMJE uniform disclosure form. The authors have no conflicts of interest to declare. 
Ethical Statement: The authors are accountable for all aspects of the work in ensuring that questions related to the accuracy or integrity of any part of the work are appropriately investigated and resolved.

\section{REFERENCES}

[1]Principi N, Di Pietro GM, Esposito S. Bronchopulmonary dysplasia: clinical aspects and preventive and therapeutic strategies[J]. J Transl Med, 2018; 16(1):36.

[2]Megan K Tracy, Sara K Berkelhamer. Bronchopulmonary Dysplasia and Pulmonary Outcomes of Prematurity[J]. Pediatr Ann, 2019; 48(4):e148-e153.

[3]Kent A Willis, Mark F Weems. Hemodynamically significant patent ductus arteriosus and the development of bronchopulmonary dysplasia[J]. Congenit Heart Dis, 2019; 14(1):27-32.

[4]Lohmann P, Luna RA, Hollister EB, et al. The airway microbiome of intubated premature infants: characteristics and changes that predict the development of bronchopulmonary dysplasia[J]. Pediatr Res, 2014; 76(3):294-301.

[5]Chen LL, Zmuda EJ, Talavera MM, et al. Dual-specificity phosphatase (DUSP) genetic variants predict pulmonary hypertension in patients with bronchopulmonary dysplasia[J]. Pediatr Res, 2020; 87(1):81-87.

[6]Moriyama G, Tanigawa M, Sakai K, et al. Synergistic effect of targeting dishevelled3 and the epidermal growth factor receptor-tyrosine kinase inhibitor on mesothelioma cells in vitro[J]. Oncol Lett, 2018; 15(1):833-838.

[7]Wang DP, Gu LL, Xue Q, et al. CtBP2 promotes proliferation and reduces drug sensitivity in non-small cell lung cancer via the Wnt/ $\beta$-catenin pathway[J]. Neoplasma, 2018; 65(6):888-897.

[8]Guan H, Zhu T, Wu S, et al. Long noncoding RNA LINC00673-v4 promotes aggressiveness of lung adenocarcinoma via activating $\mathrm{Wnt} / \beta$-catenin signaling $[\mathrm{J}]$. Proc Natl Acad Sci U S A, 2019; 116(28):14019-14028.

[9]Das B , Sinha D. Diallyl disulphide suppresses the cannonical Wnt signaling pathway and reverses the fibronectin-induced epithelial mesenchymal transition of A549 lung cancer cells[J]. Food Funct, 2019;10(1):191-202.

[10]Yves Lecarpentier, Elizabeth Gourrier, Vincent Gobert, et al. Bronchopulmonary Dysplasia: Crosstalk Between PPAR $\gamma$, Wnt $/ \beta$-Catenin and TGF- $\beta$ Pathways; The 
Potential Therapeutic Role of PPAR $\gamma$ Agonists[J]. Front Pediatr, 2019; 7:176.

[11]Jia X, Wu B, Huang J, et al. YAP and Wnt3a independently promote AECIIs proliferation and differentiation by increasing nuclear $\beta$-catenin expression in experimental bronchopulmonary dysplasia[J]. Int J Mol Med, 2021; 47(1):195-206.

[12]Sucre JMS, Deutsch GH, Jetter CS, et al. A Shared Pattern of $\beta$-Catenin Activation in Bronchopulmonary Dysplasia and Idiopathic Pulmonary Fibrosis[J]. Am J Pathol, $2018 ; 188(4): 853-862$.

[13]Y.N. Lee, Y. Gao, H.Y. Wang. Differential mediation of the Wnt canonical pathway by mammalian Dishevelleds-1, -2, and -3[J]. Cell Signal, 2008; 20(2):443-52.

[14]Zhao H, Xie C, Lin X, et al. Coexpression of IQ-domain GTPase-activating protein 1 (IQGAP1) and Dishevelled (DVL-1) is correlated with poor prognosis in non-small cell lung cancer[J]. PLoS One, 2014; 9(12):e113713.

[15]Jin C, Song P, Pang J. The CK2 inhibitor CX4945 reverses cisplatin resistance in the A549/DDP human lung adenocarcinoma cell line[J]. Oncol Lett, 2019; 18(4):3845-3856.

[16]Li Y, Sheng H, Ma F, et al. RNA m(6)A reader YTHDF2 facilitates lung adenocarcinoma cell proliferation and metastasis by targeting the AXIN1/Wnt/ $\beta$ catenin signaling[J]. Cell Death Dis, 2021; 12(5):479.

[17]Sucre JMS, Deutsch GH, Jetter CS, et al. A Shared Pattern of $\beta$-Catenin Activation in Bronchopulmonary Dysplasia and Idiopathic Pulmonary Fibrosis[J].Am J Pathol, $2018 ; 188(4): 853-862$.

[18]Lecarpentier Y, Gourrier E, Gobert V, et al. Bronchopulmonary Dysplasia: Crosstalk Between PPARy, Wnt/ $\beta$-Catenin and TGF- $\beta$ Pathways; The Potential Therapeutic Role of PPARy Agonists[J]. Front Pediatr, 2019; 7:176.

[19] Dasgupta C, Sakurai R, Wang Y, et al. Hyperoxia-induced neonatal rat lung injury involves activation of TGF- $\beta$ and Wnt signaling and is protected by rosiglitazone[J]. Am J Physiol Lung Cell Mol Physiol, 2009;296:L1031-L1041. 
Table.1 Sequences of the Real-time PCR primers.

\begin{tabular}{|c|c|c|}
\hline Gene & Primer sequences & $\begin{array}{c}\text { Product length } \\
\text { (bp) }\end{array}$ \\
\hline \multirow{2}{*}{ DVL-1 } & Forward: 5'-TCTGTACCCTGGCCCTTG $\quad-3$ ' & $142 \mathrm{bp}$ \\
\hline & Reverse: 5'-TGCTCTTGCTCCCTTCACT -3' & \\
\hline \multirow{2}{*}{$\beta$-catenin } & Forward: 5'- TATGAGTGGGAGCAAGGC -3' & $150 \mathrm{bp}$ \\
\hline & Reverse: 5'- CTGCGTGGATGGGATCT -3' & \\
\hline \multirow{2}{*}{ Cyclin D1 } & Forward: 5'- GCGTACCCTGACACCAAT-3' & 178 bp \\
\hline & Reverse: 5'-CTTCGCACTTCTGCTCCT -3' & \\
\hline \multirow[t]{2}{*}{ Ctnnbl1 } & Forward: 5'- AGGTGGTCGCACTATTGG-3' & $125 \mathrm{bp}$ \\
\hline & Reverse: 5'-GCACATCTCTGGACGGA-3' & \\
\hline \multirow[t]{2}{*}{ Gapdh } & Forward: 5'- CAAGTTCAACGGCACAGTCAAG -3' & $123 \mathrm{bp}$ \\
\hline & Reverse: 5'- ACATACTCAGCACCAGCATCAC -3' & \\
\hline
\end{tabular}


Table2 Results of immunohistochemical staining.

\begin{tabular}{|c|c|c|c|c|}
\hline & \multicolumn{2}{|c|}{$7^{\text {th }}$ day } & \multicolumn{2}{|c|}{$14^{\text {th }}$ day } \\
\hline & hyperoxia group & control group & hyperoxia group & control group \\
\hline & IHC score & IHC score & IHC score & IHC score \\
\hline \multicolumn{5}{|c|}{ Target proteins } \\
\hline$\beta$-catenin & $3.42 \pm 0.38^{* *}$ & $1.56 \pm 0.29$ & $5.26 \pm 0.92^{* *}$ & $1.54 \pm 0.37$ \\
\hline DVL-1-1 & $3.51 \pm 0.42^{* *}$ & $1.49 \pm 0.35$ & $5.84 \pm 0.89^{* *}$ & $1.48 \pm 0.76$ \\
\hline Cyclin D1 & $3.70 \pm 0.91^{* *}$ & $1.51 \pm 0.46$ & $5.78 \pm 0.84^{* *}$ & $1.69 \pm 0.86$ \\
\hline
\end{tabular}

${ }^{* *} \boldsymbol{P}<0.01$ versus control group. 
Table3 Oxidative stress on hyperoxia exposure lungs (mean \pm SD, $n=9$ )

\begin{tabular}{lcccc}
\hline Parameters & \multicolumn{3}{c}{ Groups } \\
& \multicolumn{2}{c}{ hyperoxia group } & \multicolumn{2}{c}{ control group } \\
& $7^{\text {th }}$ day & $14^{\text {th }}$ day & $7^{\text {th }}$ day & $14^{\text {th }}$ day \\
\hline MDA(mmol/g prot) & $0.71 \pm 0.16^{*}$ & $1.38 \pm 0.31^{* *}$ & $0.61 \pm 0.11$ & $0.65 \pm 0.24$ \\
SOD(U/g prot) & $24.76 \pm 3.85^{*}$ & $18.86 \pm 4.36^{* *}$ & $55.47 \pm 4.39$ & $52.92 \pm 3.96$ \\
\hline
\end{tabular}

${ }^{*} \boldsymbol{P}<0.05$ versus control group; ${ }^{* * *} \boldsymbol{P}<0.01$ versus control group. 
A

$$
7^{\text {th }} \text { day hyperoxia group control group }
$$
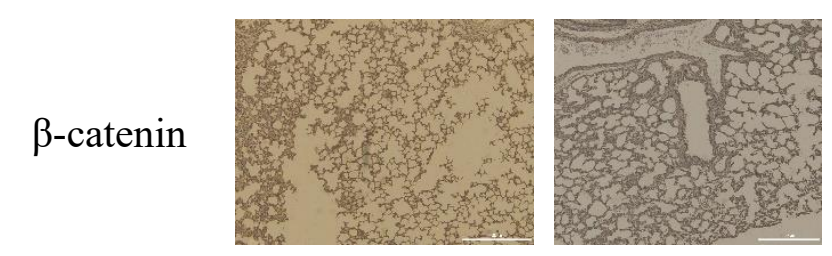

DVL-1-1
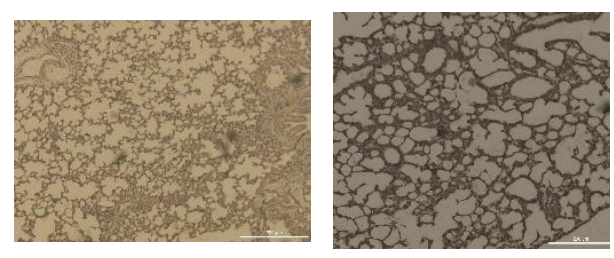

Cyclin D1

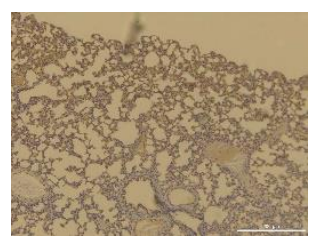

B

$14^{\text {th }}$ day hyperoxia group control group
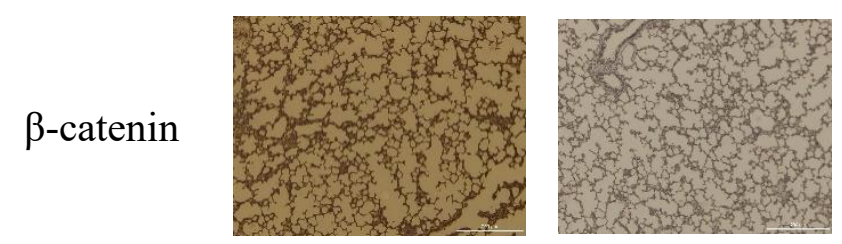

DVL-1-1
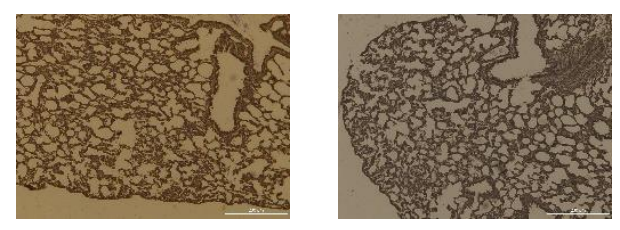

Cyclin D1

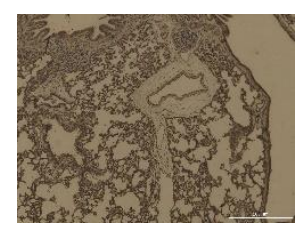

Figure 1. Hyperoxia exposure could increase the expression of B-CATENIN, DVL1 and CYCLIN D1 in the lung. 
$\mathbf{A}$

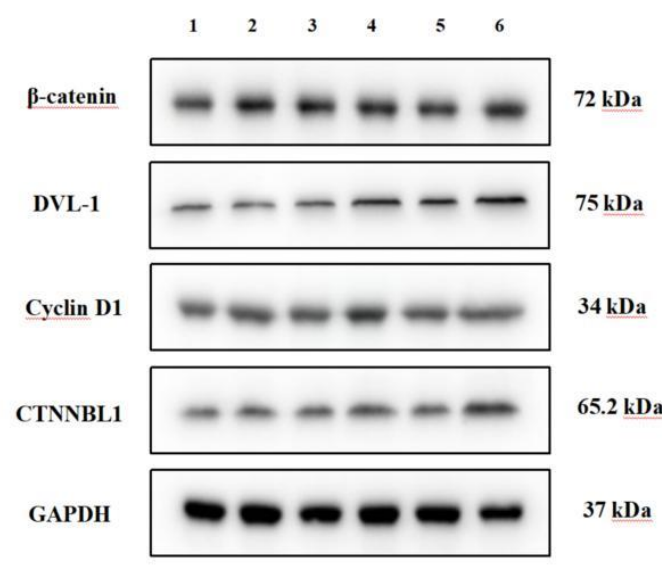

B

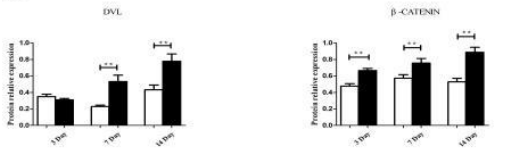

男in

圆i

C
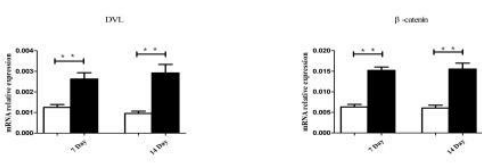

目

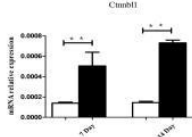

Figure 2. Hyperoxia-exposure upregulated DVL-1 protein expression and activated Wnt/ $\beta$-catenin signaling pathway in newborn rat lung.

(A) Expression of $\beta$-CATENIN, DVL-1, CTNNBL1 and CYCLIN D1 proteins levels by western blot, (B) normalized against GAPDH. (C) Expression of $\beta$-catenin, DVL-1, Ctnnbl1 and Cyclin D1 mRNA levels by real-time PCR and normalized against Gapdh, $* P<0.05, * * P<0.01$ compared with the normal group. 